\title{
Hepatitis C virus p7 induces mitochondrial depolarization of isolated liver mitochondria
}

\author{
DEOK-GYUN YOU ${ }^{1}$, HYE-RA LEE ${ }^{1,2}$, WON-KI KIM ${ }^{3}$, \\ HYUNG JUNG KIM ${ }^{4}$, GI YOUNG LEE ${ }^{1}$ and YOUNG DO YOO ${ }^{1}$ \\ ${ }^{1}$ Laboratory of Molecular Cell Biology, Graduate School of Medicine, College of Medicine; \\ ${ }^{2}$ Department of Biosystems and Biotechnology, College of Life Sciences and Biotechnology; \\ ${ }^{3}$ Department of Neuroscience, College of Medicine, Korea University, Seoul 02841; ${ }^{4}$ Department of Internal Medicine, \\ Yonsei University College of Medicine, Yonsei University Health System, Seoul 03722, Republic of Korea
}

Received December 22, 2016; Accepted March 20, 2017

DOI: $10.3892 / \mathrm{mmr} .2017 .7809$

\begin{abstract}
Hepatitis C virus (HCV)-encoded protein p7 is a viroporin that acts as an ion channel and is indispensable for $\mathrm{HCV}$ particle production. Although the main target of HCV p7 is the endoplasmic reticulum, it also targets mitochondria. $\mathrm{HCV}$-infected cells show mitochondrial depolarization and ATP depletion; however, the function of HCV p7 in mitochondria is not fully understood. The present study demonstrated that treatment of isolated mouse liver mitochondria with the synthesized HCV p7 protein induced mitochondrial dysfunction. It also demonstrated that $\mathrm{HCV}$ p7 targeted isolated mouse liver mitochondria and induced mitochondrial depolarization. In addition, $\mathrm{HCV}$ p7 triggered matrix acidification and, ultimately, a decrease in ATP synthesis in isolated mitochondria. These findings indicate that targeting of mitochondria by $\mathrm{HCV}$ p7 in infected cells causes mitochondrial dysfunction to support HCV particle production. The present study provided evidence for the role of $\mathrm{HCV}$ p7 in mitochondria, and may lead to the development of novel strategies for HCV therapy.
\end{abstract}

Correspondence to: Professor Young Do Yoo or Dr Gi Young Lee, Laboratory of Molecular Cell Biology, Graduate School of Medicine, College of Medicine, Korea University, 73 Inchon-ro, Seongbuk-gu, Seoul 02841, Republic of Korea

E-mail: ydy1130@korea.ac.kr

E-mail: gylative@gmail.com

Abbreviations: $\Delta \Psi \mathrm{m}, \quad$ mitochondrial membrane potential; ADP, adenosine 5'-diphosphate; BCECF-AM, 2',7'-bis-(2carboxyethyl)-5-(and-6)-carboxyfluorescein, acetoxymethyl ester; BSA, bovine serum albumin; CCCP, carbonyl cyanide 3-chlorophenylhydrazone; EGTA, ethylene glycol-bis (2-aminoet hylether)-N,N,N',N'-tetraacetic acid; FSC, forward scatter; HCC, hepatocellular carcinoma; $\mathrm{HCV}$, hepatitis $\mathrm{C}$ virus; $\mathrm{SSC}$, side scatter, TFE, trifluoroethanol

Key words: hepatitis, hepatitis $\mathrm{C}$ virus p7, mitochondria, mitochondrial dysfunction, mitochondrial depolarization

\section{Introduction}

Hepatitis $\mathrm{C}$ virus (HCV) is one of the common factors associated with development of chronic liver inflammation and liver disease, a major problem in global health. HCV causes not only hepatitis, but also a variety of diseases including liver fibrosis, cirrhosis and hepatocellular carcinoma (HCC) (1). $\mathrm{HCV}$ is a single-stranded RNA virus that encodes several polyproteins; three structural proteins (core, E1, and E2) and seven non-structural proteins (p7, NS2, NS3, NS4A, NS4B, NS5A and NS5B) (2). It has been reported that these structural proteins constitute the HCV viral particle, and the non-structural proteins serve a variety of roles in the HCV life cycle (2). However, the exact function and mechanism of HCV polyproteins are not fully understood.

One of the non-structural proteins, $\mathrm{p} 7$, has been reported to be a small hydrophobic protein that not only interacts with a variety of biological membranes (3), but also has ion channel activity as a viroporin (4). p7 is localized to a variety of cellular organelles, including the endoplasmic reticulum and plasma membrane $(3,5)$. A previous study demonstrated that p7 is also localized in mitochondria (4). This variety of subcellular localizations suggests that $\mathrm{p} 7$ has diverse physiological roles in hepatocytes. However, to date, the function of $\mathrm{p} 7$ has been mainly discussed with regard to the viral life cycle; for example, the assembly or release of viral particles $(6,7)$. In particular, the correlation between $\mathrm{p} 7$ and mitochondrial function has not yet been fully elucidated.

Mitochondria are the central organelles of cells. Since mitochondria supply the majority of the cellular energy by oxidative phosphorylation, they serve a key role in energy metabolism in cells. For this reason, mitochondria are often called the 'powerhouses' of cells (8). Therefore, mitochondrial function is one of the most important factors for cellular function, and the mitochondrial membrane potential $(\Delta \Psi \mathrm{m})$ is a major determinant of the fate of mitochondria and cells (9). Several studies have suggested that viral infection affects mitochondrial functions, and in particular is correlated with mitochondrial depolarization (10-14). It has been reported that certain viroporins, including human T-cell leukemia virus type 1 (HTLV-1) p13 and picornavirus 2B, can be localized on 
mitochondria and cause mitochondrial depolarization $(15,16)$. However, the exact molecular mechanisms of viral infection-induced mitochondrial dysfunction and changes in cell metabolism remain to be elucidated.

A number of studies into $\mathrm{HCV}$ infection have previously reported that $\mathrm{HCV}$ infection is associated with mitochondrial dysfunction in hepatocytes, and it has been documented that the HCV core protein alters mitochondrial functions in Huh7 and Huh7.5 cells $(10,13,17)$. However, to the best of the authors' knowledge, there is no direct evidence of the association between $\mathrm{p} 7$ and liver mitochondria. Therefore, the purpose of the present study was to investigate the exact role of $\mathrm{p} 7$ in mitochondrial function. To this end, isolated mouse liver mitochondria and synthesized $\mathrm{p} 7$ protein were used in order to investigate the effects of p7.

\section{Materials and methods}

Chemicals. Dimethyl sulfoxide, adenosine 5'-diphosphate (ADP), D-mannitol, sucrose, rotenone, ethylene glycol-bis (2-aminoethylether)-N,N,N',N'-tetraacetic acid (EGTA), 4-(2-hydroxyethyl) piperazine-1-ethanesulfonic acid, magnesium chloride $\left(\mathrm{MgCl}_{2}\right)$, n-(2-hydroxyethyl) piperazine-n'-2-ethanesulfonic acid) (HEPES), trifluoroethanol (TFE), 2',7'-bis-(2-carboxyethyl)-5-(and-6)-carboxyfluorescein, acetoxymethyl ester (BCECF-AM), carbonyl cyanide 3-chlorophenylhydrazone (CCCP) and bovine serum albumin (BSA) were all purchased from Sigma-Aldrich; Merck KGaA (Darmstadt, Germany). Anhydrous monobasic potassium phosphate, $\left(\mathrm{KH}_{2} \mathrm{PO}_{4}\right)$, Triton $\mathrm{X}-100$, 5-, 5'-,6-,6'-tetrachloro-1,1', 3,3' tetraethyl benzimidazolyl carbocyanine iodide/chloride (JC-1; M34152), MitoTracker Green FM (M7514), and an ATP determination kit (A22066) were purchased from Thermo Fisher Scientific, Inc. (Waltham, MA, USA). The HCV p7 amino acid sequence (genotype 1a, H77 strain) is ALENLVI LNA ${ }^{10}$ ASLAGTHGLV ${ }^{20}$ SFLVFFCFAW $^{30}$ YLKGRWVPGA ${ }^{40}$ VYAFYGMWPL ${ }^{50}$ LLLLLALPQR $^{60}$ AYA. 5-carboxy-tetramethylrhodamine (TAMRA)-p7 (HCV genotype 1a) was chemically synthesized by GL Biochem (Shanghai, China) and dissolved in TFE.

Animals. The experimental procedures were approved by the Institutional Animal Care and Use Committee of the Korea University College of Medicine (KOREA-2016-0174). Adult male C57 BL/6 N mice (6 weeks old; weight, 16-21 g) were purchased from Orient Bio Inc. (Gyenggi-do, South Korea). Mice were housed in regulated conditions $\left(21 \pm 2^{\circ} \mathrm{C} ; 50 \pm 5 \%\right.$ humidity; $12 \mathrm{~h} / 12 \mathrm{~h}$ light/dark cycle with light on from $8 \mathrm{AM}$ ) with free access to food and water.

Isolation of mitochondria. All isolation steps were performed on ice or using ice-cold isolation buffers (IB: $225 \mathrm{mM}$ mannitol, $75 \mathrm{mM}$ sucrose and $30 \mathrm{mM}$ Tris- $\mathrm{HCl}, \mathrm{pH}$ 7.4; IB-1: $0.5 \%$ BSA and $0.5 \mathrm{mM}$ EGTA in IB; IB-2: $0.5 \%$ BSA in IB) and the mitochondrial sample was prepared as described in (18). Briefly, following the decapitation of the mice, the livers $(0.8 \mathrm{~g} \pm 5 \%)$ were washed with IB buffer and cut into small pieces. Liver tissue was homogenized with a glass-tissue grinder (Wheaton Industries Inc., Millville, NJ, USA) in IB-1 buffer, and crude mitochondrial fractions were isolated from suspensions by density-gradient centrifugation (10,000 x $\mathrm{g}$ at $4^{\circ} \mathrm{C}$ ) for $10 \mathrm{~min}$. Mitochondrial pellets were resuspended in mitochondrial resuspension buffer [MRB: $250 \mathrm{mM}$ mannitol, $5 \mathrm{mM}$ HEPES (pH 7.4) and 0.5 mM EGTA]. All the following experiments were performed within $9 \mathrm{~h}$ from the time of mitochondria isolation.

Flow cytometric analysis. Flow cytometric assays to measure $\Delta \Psi \mathrm{m}$ and mitochondrial matrix acidification were performed using the FACSCalibur (BD Biosciences, San Jose, CA, USA) and data were analyzed with FlowJo software version 10.0.7 (Tree Star, Inc., Ashland, OR, USA). Mitochondrial samples were gated according to side scatter and forward scatter to optimize mitochondrial selection. For each sample, $\sim 300$ events/sec were counted, up to 20,000 counts. Isolated mitochondrial samples were incubated in a buffer composed of $250 \mathrm{mM}$ mannitol, $5 \mathrm{mM}$ HEPES (pH 7.4), $1 \mathrm{mM} \mathrm{KH}_{2} \mathrm{PO}_{4}$, $10 \mu \mathrm{M}$ EGTA and $2 \mu \mathrm{M}$ rotenone at room temperature.

Confocal microscopy of isolated mouse liver mitochondria. Isolated mitochondria were incubated with $0.1 \mu \mathrm{M}$ TAMRA-p7 for 5 min to target mitochondria and stained with $0.1 \mu \mathrm{M}$ MitoTracker Green FM for 5 min at room temperature. The samples were loaded onto a cover glass with a confocal dish, and confocal microscopy was performed in a dedicated room at a constant temperature $\left(24 \pm 2^{\circ} \mathrm{C}\right)$ and humidity $(\sim 40 \%)$. The data were acquired using a Zeiss LSM 700 microscope (Zeiss GmbH, Jena, Germany).

Spectrophotometric assay of $\Delta \Psi m$. Mitochondrial samples were treated with $\mathrm{p} 7$ protein $(1 \mu \mathrm{g} / \mathrm{ml})$ for $5 \mathrm{~min}$ and then incubated with JC-1 $(1 \mu \mathrm{M})$ for $10 \mathrm{~min}$ and transferred to black 96-well plates (Optiplate-96F; PerkinElmer, Inc., Waltham, MA, USA) for the measurement of fluorescence at $488 \mathrm{~nm}$ excitation. The fluorescence was detected at emission wavelengths of $525 \mathrm{~nm}$ (monomers) and $594 \mathrm{~nm}$ (aggregates). The mitochondrial membrane potentials were measured using a microplate reader (Molecular Devices, LLC, Sunnyvale, CA, USA).

ATP assay. For the ATP assay, $10 \mu \mathrm{M}$ ADP and $5 \mathrm{mM}$ succinate were added to the mitochondrial samples $(8 \mu \mathrm{g} / \mathrm{ml})$ for $10 \mathrm{~min}$ at room temperature. The samples were subsequently incubated with ATP assay mix (Thermo Fisher Scientific, Inc.) for $15 \mathrm{~min}$, and ATP was measured using a GloMax 20/20 luminometer (Promega Corporation, Madison, WI, USA). ATP amounts were calculated from an ATP standard curve.

\section{Results}

The p 7 protein targets isolated mouse liver mitochondria. Previous studies have demonstrated that 7 localizes to mitochondria (5); however, the exact role of p7 in mitochondria is difficult to clarify due to the mixed activity of various $\mathrm{HCV}$-encoded proteins. Therefore, an experimental system was designed using isolated mouse liver mitochondria and synthesized p7 protein to clarify its function in mitochondria. First, a flow cytometric assay was performed with a mitochondria-sensitive probe (MitoTracker Green FM) to check the quality of isolated mitochondria. As demonstrated 
A

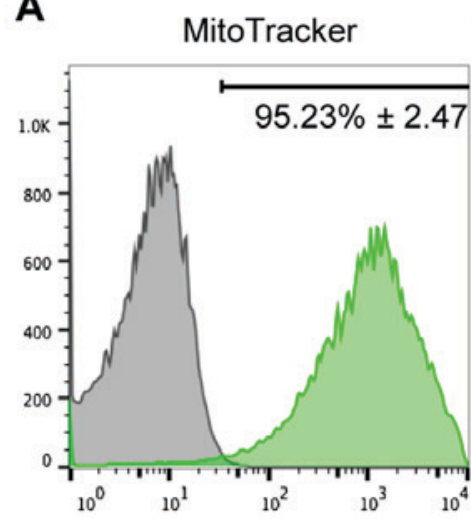

B

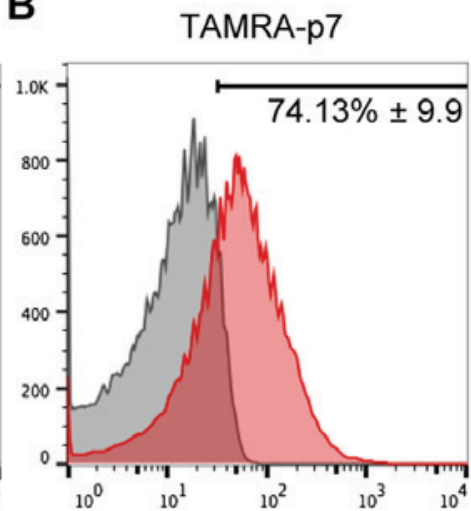

\section{C}

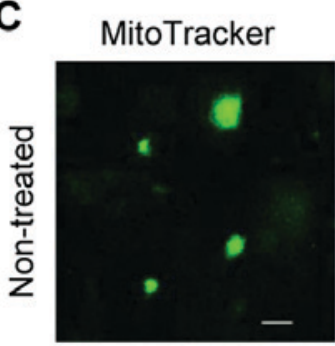

TAMRA

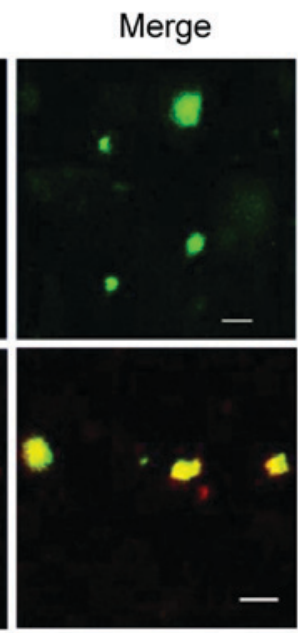

Figure 1. Mitochondrial targeting of TAMRA-p7 in isolated mouse liver mitochondria. (A) A flow cytometric assay was performed with $0.2 \mu \mathrm{M}$ MitoTracker Green FM to confirm the isolation of mitochondria. p7 targeting of mitochondria was analyzed using a flow cytometric assay with (B) $0.5 \mu \mathrm{M}$ TAMRA-p7. The data in (A and B) represent the non-treated experiment (gray) or treatments with MitoTracker-Green FM (green) or TAMRA-p7 (red). The mean \pm standard deviation is shown for four independent experiments in (A and B). (C) MitoTracker-Green FM (green), or TAMRA-p7 (red) targeted the isolated mitochondria, as observed by confocal microscopy (scale bars, $5 \mu \mathrm{m}$ ). TAMRA, 5-carboxy-tetramethylrhodamine.

in Fig. 1A, the proportion of mitochondria in the prepared sample was $>90 \%$. Next, the mitochondria were treated with the synthesized $\mathrm{p} 7$ protein. To visualize the $\mathrm{p} 7$ protein, p7 labeled with TAMRA was used and the mitochondrial targeting of $\mathrm{p} 7$ examined by flow cytometry. As expected, p7 was detected in mitochondria and the targeting efficiency was $\sim 75 \%$ (Fig. 1B). p7 targeting to mitochondria was also confirmed using confocal laser microscopy (Fig. 1C).

p7 induces mitochondrial depolarization. The $\Delta \Psi \mathrm{m}$ is an important factor determining the fate of the mitochondria, and therefore of the cells. It has previously been demonstrated that HCV infection of hepatoma cells reduces $\Delta \Psi \mathrm{m}(10,13,17)$. However, the role of $\mathrm{p} 7$ in mitochondrial dysfunction in cells infected by $\mathrm{HCV}$ has not been reported. To address this, whether $\mathrm{p} 7$ affected the $\Delta \Psi \mathrm{m}$ in isolated mitochondria from normal hepatocytes was tested. To investigate the direct effect of p7 in mitochondria, the mitochondria were treated with $\mathrm{p} 7$ protein, and the $\Delta \Psi \mathrm{m}$ change was examined using a mitochondria-sensitive fluorescent probe (JC-1), which is an indicator of mitochondrial membrane potential. First, the time for JC-1 staining to measure the mitochondrial membrane potential was optimized. As demonstrated in
Fig. 2A, the intensity ratio of red/green fluorescence reached a maximum level within $10 \mathrm{~min}$. p7 treatment reduced the $\Delta \Psi \mathrm{m}$ in a concentration-dependent manner (Fig. 2B). Fig. $2 \mathrm{C}$ shows the quantified data of Fig. $2 \mathrm{~B}$ by representing the ratio of the signal of high $\Delta \Psi \mathrm{m}$ to the signal of low $\Delta \Psi \mathrm{m}$. To confirm this result, $\Delta \Psi \mathrm{m}$ was also examined using a spectrophotometric assay. As demonstrated in Fig. 2D, p7 noticeably induced mitochondrial depolarization. CCCP was used as a positive control (Fig. 2B-D).

p7 induces mitochondrial matrix acidification. The proton motive force between the mitochondrial matrix and intermembrane space is determined predominantly by the $\Delta \Psi \mathrm{m}$ in mitochondria, and to a lesser extent, by the hydrogen ion $\left(\mathrm{H}^{+}\right)$ concentration gradient (9). These two components contribute to the electrochemical proton gradient and are important for mitochondrial function. Therefore, changes in mitochondrial matrix $\mathrm{pH}$ were examined using the fluorescence indicator, BCECF-AM $(19,20)$. To determine the optimal staining duration for BCECF-AM in isolated mitochondria, flow cytometric assays with BCECF-AM were performed. As demonstrated in Fig. 3A, the mitochondrial samples were saturated by BCECF-AM fluorescence within $10 \mathrm{~min}$; this 
A
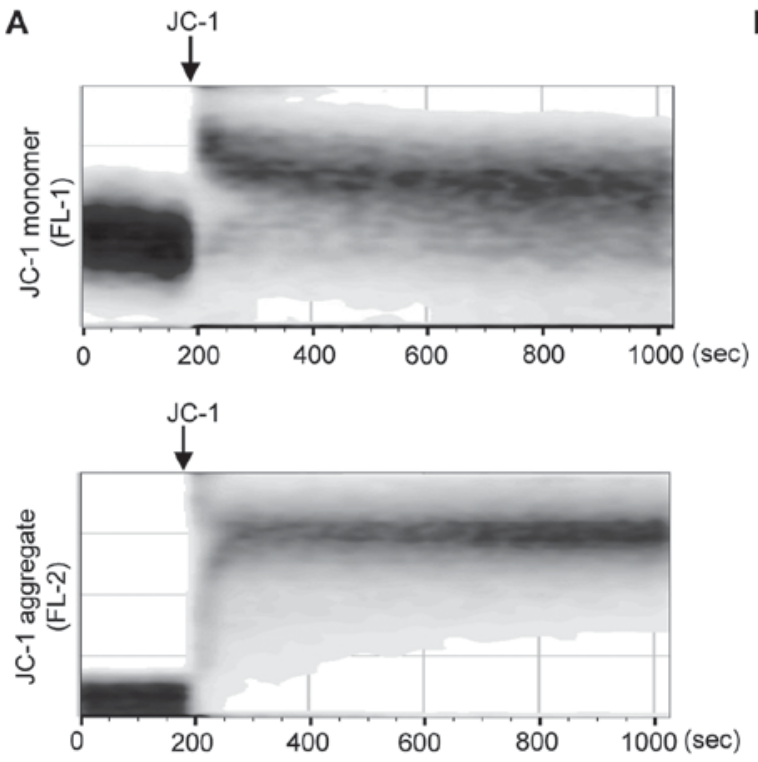

B

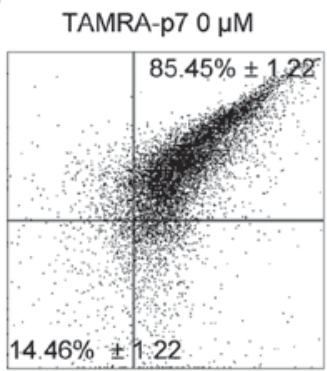

TAMRA-p7 $0.5 \mu \mathrm{M}$

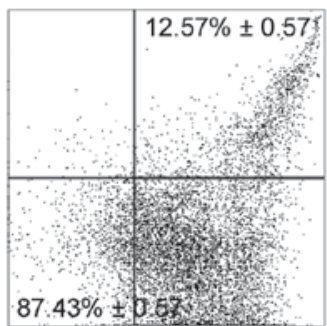

D

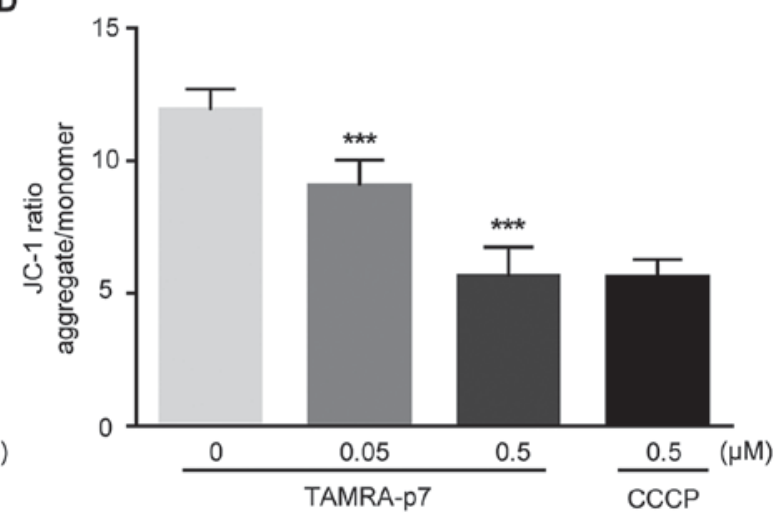

Figure 2. Effect of $\mathrm{p} 7$ on mitochondrial depolarization. (A) Mitochondrial samples were stained with $1 \mu \mathrm{M} \mathrm{JC}-1$ for time-course analysis by flow cytometry. Isolated mitochondria were treated with TAMRA-p7 for $5 \mathrm{~min}$ and stained with JC-1 for $10 \mathrm{~min}$. (B) Experimental data were quantified using (C) flow cytometry analysis and (D) a spectrophotometric assay. CCCP was used as a positive control. The data represent the mean of three independent experiments. ${ }^{* *} \mathrm{P} \leq 0.01$ and ${ }^{* * *} \mathrm{P} \leq 0.001$ vs. control by two-way analysis of variance. JC-1, 5-,5'-,6-,6'-tetrachloro-1,1',3,3' tetraethyl benzimidazolyl carbocyanine iodide/chloride; TAMRA, 5-carboxy-tetramethylrhodamine; CCCP, carbonyl cyanide 3-chlorophenylhydrazone.

indicated a stable $\mathrm{pH}$ level of the isolated mitochondria. Matrix acidification triggered by $\mathrm{p} 7$ was examined by flow cytometric analysis in mitochondrial samples, and this revealed that the $\mathrm{pH}$ of the mitochondrial matrix was decreased by $\mathrm{p} 7$ treatment in a concentration-dependent manner (Fig. 3B and C). These results demonstrated that $\mathrm{p} 7$ caused acidification of the mitochondrial matrix.

$p 7$ decreases mitochondrial ATP production. The findings above demonstrated that $\mathrm{p} 7$ induced mitochondrial depolarization and mitochondrial matrix acidification. Subsequently, the effect of $\mathrm{p} 7$ on mitochondrial ATP production was measured, since $\Delta \Psi \mathrm{m}$ is known to be the driving force for ATP synthesis, and an electrochemical hydrogen ion gradient also contributes to the ATP synthesis process (9). As expected, ATP production was decreased by $\mathrm{p} 7$ treatment in a concentration-dependent manner (Fig. 4). On the basis of results obtained in the present study, it is suggested that the p7 protein directly targets mitochondria to induce mitochondrial dysfunction.

\section{Discussion}

Previous studies have reported that disruption of $\Delta \Psi \mathrm{m}$ was induced by HCV infection in Huh7.5 cells, and that HCV infection affected mitochondrial functions, including the redox system, calcium signaling and ATP generation $(12,14,21)$. To date, the involvement of HCV p7 in mitochondrial dysfunction has been not reported, although p7 is known to target to mitochondria (5). Therefore, the aim of the present study was to investigate the direct effects of $\mathrm{p} 7 \mathrm{in}$ mitochondria. To minimize the contribution of indirect effects of $\mathrm{p} 7$ in mitochondria, purified mitochondria from mouse liver and synthesized $\mathrm{p} 7$ protein were used. It was identified that $\mathrm{p} 7$ induced mitochondrial depolarization (Fig. 2), matrix acidification (Fig. 3) and decreased ATP synthesis (Fig. 4), clearly demonstrating that $\mathrm{p} 7$ serves a direct role in mitochondrial dysfunction. Other HCV-encoded proteins, including the core protein and NS5A, are also associated with mitochondria and affect mitochondrial dysfunction $(10,13)$. It was difficult to demonstrate that $\mathrm{p} 7$ was a major factor in $\mathrm{HCV}$ 

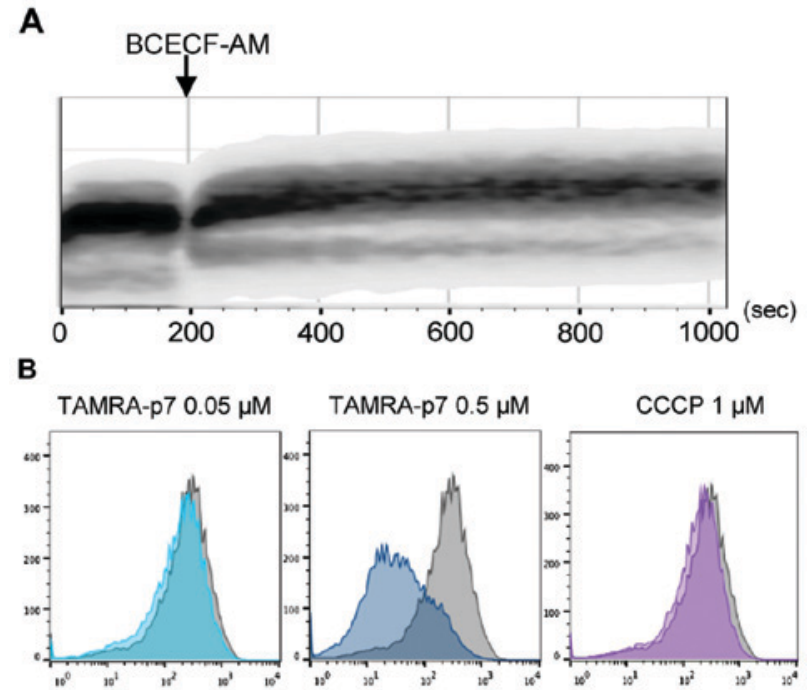

C

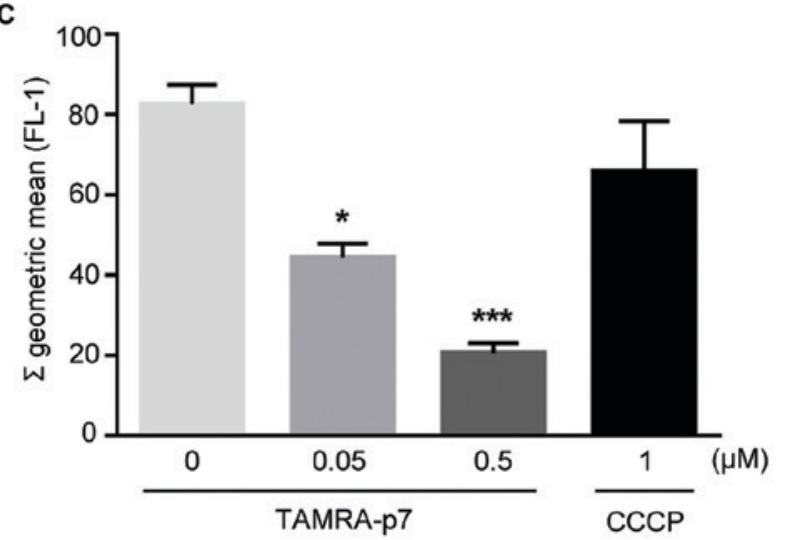

Figure 3. Mitochondrial acidification induced by p7. (A) The mitochondrial samples were stained with $1 \mu \mathrm{M}$ BCECF-AM for time-course analysis by flow cytometry. (B) Isolated mitochondria were treated with TAMRA-p7 for 5 min and stained with BCECF-AM for $10 \mathrm{~min}$. (C) Experimental data were quantified with flow cytometry. CCCP was used as a positive control. The data represent the mean of three independent experiments. " $\mathrm{P} \leq 0.05$ and ${ }^{* * *} \mathrm{P} \leq 0.001$ vs. control by two-way analysis of variance. BCECF-AM, 2',7'-bis-(2-carboxyethyl)-5-(and-6)-carboxyfluorescein, acetoxymethyl ester; TAMRA, 5-carboxy-tetramethylrhodamine; CCCP, carbonyl cyanide 3-chlorophenylhydrazone.

infection-induced mitochondrial dysfunction. Nonetheless, it has been demonstrated that p7 specifically targeted the purified mitochondria, and had a clear effect on mitochondrial function.

The results of the present study demonstrated that p7 treatment caused mitochondrial matrix acidification (Fig. 3). HCV p7 has been reported to function as a proton channel (22), and thus could affect mitochondrial matrix acidification. However, it was assumed that p7-induced mitochondrial matrix acidification was a small aspect of p7-induced mitochondrial depolarization, since p7 may also conduct other cations, including sodium and potassium ions. It is suggested that p7-induced mitochondrial depolarization is the sum of ion gradient dissipation of protons and a potassium gradient.

It was also demonstrated that p7 affected ATP synthesis in mitochondria (Fig. 4). The results of the present study are in accord with those reported in earlier experimental studies in hepatocytes $(14,23,24)$. To the best of the authors'

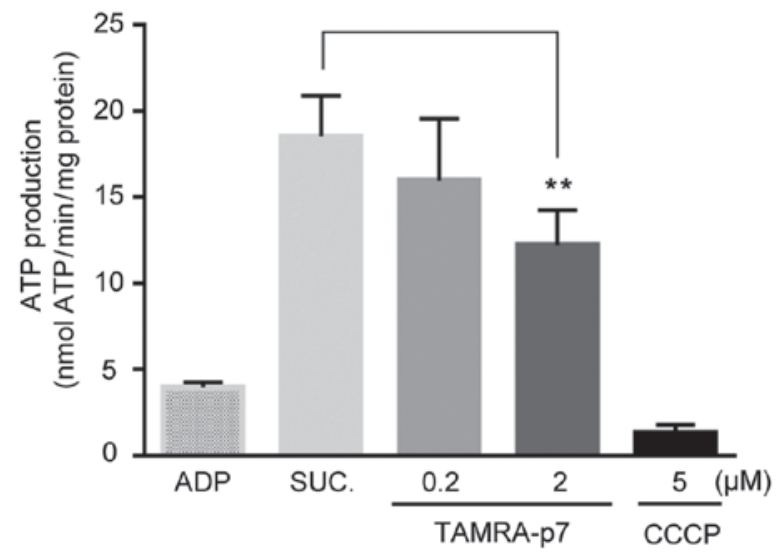

Figure 4. Effect of p7 on mitochondrial ATP synthesis. Isolated mitochondria were incubated with $\mathrm{p} 7$, and then $\operatorname{ADP}(10 \mu \mathrm{M})$ and succinate $(5 \mathrm{mM})$ were added to supply substrates for mitochondrial ATP synthesis. The graph indicates decreased ATP synthesis in a p7 concentration-dependent manner. CCCP was used as a positive control. The data shown are the mean of three independent experiments. ${ }^{* *} \mathrm{P} \leq 0.01$ vs. control by two-way analysis of variance. ATP, adenosine triphosphate; ADP, adenosine 5'-diphosphate; CCCP, carbonyl cyanide 3-chlorophenylhydrazone; SUC., succinate.

knowledge, however, this is the first demonstration of a direct association between $\mathrm{HCV}$ p7 and mitochondria. Nevertheless, the phenomenon of HCV infection-induced ATP depletion remains controversial. Normally, the energy requirement increases in virus-infected cells for synthesis of the viral proteins, whereas p7-induced mitochondrial depolarization and the proton gradient dissipation lead to a mitochondrial energy crisis. It has been reported that certain viral infections induce a significant decrease in ATP concentration in hepatoma cell lines (16). In addition, these viral proteins induce mitochondrial disruptions and, in certain cases, cellular death. Since p7 serves an important role in HCV maturation and budding, but not RNA replication, this phenomenon could be explained by the concept of a 'viral budding strategy' to induce mitochondria-mediated apoptosis at late stages of HCV infection, including the viral budding process.

In conclusion, the present study suggested that $\mathrm{HCV}$ p7 serves an important role in mitochondrial dysfunction. The findings have enhanced our understanding of the function of p7 in mitochondria. Although further studies are required to determine the exact mechanism and topology of $\mathrm{p} 7$ in the mitochondrial membrane, the findings of the present study may aid the development of future HCV therapies.

\section{Acknowledgements}

The present study was supported by the National Research Foundation of Korea grant funded by the Korean government (grant no. NRF-2013R1A1A2063171), by Basic Science Research Program through the National Research Foundation funded by the Ministry of Education, Science and Technology (grant no. NRF-2013R1A1A2009792), by Basic Science Research Program through the National Research Foundation (NRF) funded by the Ministry of Education (grant no. NRF-2017R1D1A1B03032322), and by a grant from the Korea Health Technology R\&D Project (grant no. HI15C2796) of the 
Korea Health Industry Development Institute, which is funded by the Ministry of Health and Welfare, Republic of Korea.

\section{References}

1. National Institutes of Health: National Institutes of Health Consensus Development Conference Statement: Management of hepatitis C: 2002-June 10-12, 2002. Hepatology 36 (5 Suppl 1): S3-S20, 2002.

2. Suzuki T, Ishii K, Aizaki H and Wakita T: Hepatitis C viral life cycle. Adv Drug Deliv Rev 59: 1200-1212, 2007.

3. Carrere-Kremer S, Montpellier-Pala C, Cocquerel L, Wychowski C, Penin F and Dubuisson J: Subcellular localization and topology of the p7 polypeptide of hepatitis $\mathrm{C}$ virus. J Virol 76: 3720-3730, 2002.

4. Griffin SD, Harvey R, Clarke DS, Barclay WS, Harris M and Rowlands DJ: A conserved basic loop in hepatitis $\mathrm{C}$ virus p7 protein is required for amantadine-sensitive ion channel activity in mammalian cells but is dispensable for localization to mitochondria. J Gen Virol 85: 451-461, 2004.

5. Griffin S, Clarke D, McCormick C, Rowlands D and Harris M Signal peptide cleavage and internal targeting signals direct the hepatitis $\mathrm{C}$ virus $\mathrm{p} 7$ protein to distinct intracellular membranes J Virol 79: 15525-15536, 2005.

6. Sakai A, Claire MS, Faulk K, Govindarajan S, Emerson SU, Purcell RH and Bukh J: The p7 polypeptide of hepatitis C virus is critical for infectivity and contains functionally important genotype-specific sequences. Proc Natl Acad Sci USA 100 11646-11651, 2003.

7. Steinmann E, Penin F, Kallis S, Patel AH, Bartenschlager R and Pietschmann T: Hepatitis C virus p7 protein is crucial for assembly and release of infectious virions. PLoS Pathog 3: e103, 2007.

8. McBride HM, Neuspiel M and Wasiak S: Mitochondria: More than just a powerhouse. Curr Biol 16: R551-R560, 2006.

9. Dzbek J and Korzeniewski B: Control over the contribution of the mitochondrial membrane potential (DeltaPsi) and proton gradient (DeltapH) to the protonmotive force (Deltap). In silico studies. J Biol Chem 283: 33232-33239, 2008.

10. Okuda M, Li K, Beard MR, Showalter LA, Scholle F, Lemon SM and Weinman SA: Mitochondrial injury, oxidative stress and antioxidant gene expression are induced by hepatitis $\mathrm{C}$ virus core protein. Gastroenterology 122: 366-375, 2002.

11. Boya P, Pauleau AL, Poncet D, Gonzalez-Polo RA, Zamzami N and Kroemer G: Viral proteins targeting mitochondria: Controlling cell death. Biochim Biophys Acta 1659: 178-189, 2004
12. Piccoli C, Scrima R, D'Aprile A, Ripoli M, Lecce L, Boffoli D and Capitanio N: Mitochondrial dysfunction in hepatitis $C$ virus infection. Biochim Biophys Acta 1757: 1429-1437, 2006.

13. Wang T, Campbell RV, Yi MK, Lemon SM and Weinman SA: Role of hepatitis $C$ virus core protein in viral-induced mitochondrial dysfunction. J Viral Hepat 17: 784-793, 2010.

14. Brault C, Levy PL and Bartosch B: Hepatitis C virus-induced mitochondrial dysfunctions. Viruses 5: 954-980, 2013.

15. Silic-Benussi M, Marin O, Biasiotto R, D'Agostino DM and Ciminale V: Effects of human T-cell leukemia virus type 1 (HTLV-1) p13 on mitochondrial K+ permeability: A new member of the viroporin family? FEBS Lett 584: 2070-2075, 2010.

16. Su YC and Hong JR: Betanodavirus B2 causes ATP depletion-induced cell death via mitochondrial targeting and complex II inhibition in vitro and in vivo. J Biol Chem 285: 39801-39810, 2010.

17. Deng L, Adachi T, Kitayama K, Bungyoku Y, Kitazawa S, Ishido S, Shoji I and Hotta H: Hepatitis $\mathrm{C}$ virus infection induces apoptosis through a Bax-triggered, mitochondrion-mediated, caspase 3-dependent pathway. J Virol 82: 10375-10385, 2008.

18. Wieckowski MR, Giorgi C, Lebiedzinska M, Duszynski J and Pinton P: Isolation of mitochondria-associated membranes and mitochondria from animal tissues and cells. Nat Protoc 4: 1582-1590, 2009

19. Brierley GP, Davis MH, Cragoe EJ and Jung DW: Kinetic properties of the sodium/hydrogen ion antiport of heart mitochondria. Biochemistry 28: 4347-4354, 1989.

20. Jung DW, Davis MH and Brierley GP: Estimation of matrix pH in isolated heart mitochondria using a fluorescent probe. Anal Biochem 178: 348-354, 1989.

21. Tardif KD, Waris G and Siddiqui A: Hepatitis C virus, ER stress, and oxidative stress. Trends Microbiol 13: 159-163, 2005.

22. StGelais C, Foster TL, Verow M, Atkins E, Fishwick CW, Rowlands D, Harris M and Griffin S: Determinants of hepatitis $\mathrm{C}$ virus $\mathrm{p} 7$ ion channel function and drug sensitivity identified in vitro. J Virol 83: 7970-7981, 2009.

23. Piccoli C, Scrima R, Quarato G, D'Aprile A, Ripoli M, Lecce L, Boffoli D, Moradpour D and Capitanio N: Hepatitis C virus protein expression causes calcium-mediated mitochondrial bioenergetic dysfunction and nitro-oxidative stress. Hepatology 46: 58-65, 2007.

24. Ando T, Imamura H, Suzuki R, Aizaki H, Watanabe T, Wakita T and Suzuki T: Visualization and measurement of ATP levels in living cells replicating hepatitis $\mathrm{C}$ virus genome RNA. PLoS Pathog 8: e1002561, 2012. 